\title{
Self-Adaptive Nonlinear Least Squares Optimization for Geometric Calculations
}

\author{
Alpar A. Csendes \\ GRAPHISOFT SE, Záhony u. 7. (GRAPHISOFT Park 1.) 1031 Budapest, Hungary
}

How to cite this paper: Alpar A. Csendes. (2020) Self-Adaptive Nonlinear Least Squares Optimization for Geometric Calculations. Journal of Applied Mathematics and Computation, 4(1), 5-13.

DOI: $10.26855 /$ jamc. 2020.03 .002

Received: May 25, 2019

Accepted: June 22, 2019

Published: March 6, 2020

*Corresponding author: Alpar A. Csendes. GRAPHISOFT SE, Záhony u. 7. (GRAPHISOFT Park 1.) 1031 Budapest, Hungary.

E-mail: $\underline{\text { ACsendes@graphisoft.com }}$

\begin{abstract}
In this paper, two-dimensional parameterized geometries and related computational techniques are discussed. Simple and extendable parameterized computational model has been established, which provides the foundation for morphing the geometry. The well known Nonlinear Least Squares optimization technique has been applied in order to find the particular shape of the geometry satisfying a given set of criteria. This criteria-system is provided by the end-user, and so it has been fundamental to provide a meaningful technique as part of the setup. Our "dimension tool" was not only the right choice for our clients (Architects) but also the related mathematical model proved to be easily handled by the numerical solution process. The solution is found via minimization of the sum of properly weighted and squared residuals. This system of residuals has been extended by auxiliary residuals and related terms implementing an optimizationembedded continuation-technique, a new and promising approach to support stable and quick solution for stiff residual systems. At the end of the paper we show that the computational model and techniques are capable of supporting extraction of parametric sensitivity of the final geometry, which has great practical importance in design optimization.
\end{abstract}

\section{Keywords}

2D Geometry, Nonlinear Problems, Adaptive Solution Process, Geometry Sensitivities

\section{Introduction}

In computer aided design process engineers are often facing the challenge of modelling several variations of essentially the same geometry all over again to satisfy slightly different design criteria or in the process of exploring alternative designs. Often during this process, the original (master or nominal) geometry is copied and slightly modified. This is tedious work, often performed manually, and is only a workaround necessitated by the lack of proper support for parametric geometrical modelling. It is also an inherently dangerous design methodology. Imagine, that late in the design process the need for some general change in the master-geometry arises when already many copies of it exist. In such situation the engineer needs to apply changes manually to all the copies. This is not only a waste of engineering resources, but an error-prone process as well.

In order to speed up and make the design process more flexible, the development team at GRAPHISOFT SE decided to introduce parameterization of our existing profile-geometry in ArchiCAD -our software product for architectural design-. Profile geometry is essentially a two-dimensional geometry consisting of 2D shapes defined by boundary contours and holes. The contours are constructed as loops of straight edges and circular arcs connecting via vertices at the ends of each segment. We have envisioned a system tailored for architects, so our newly introduced parameters must have had straightforward visual or graphical interpretations. For this reason, our parameters are directly related to various distances of the master or nominal geometry, which is quite natural for architects, but surely also for engineers 
of other disciplines. Simply, these parameters are distance values and any change in these parameter values induce a new, modified version of the nominal geometry, called an instance. The user can change the parameter values, or -in special cases- they are controlled by automatisms already built into the software to support different architectural constraints not discussed in this paper. The nominal geometry along with the user-provided parameter definitions form the so-called parametric model. These models are stored in a library and accessed with a special editor/viewer. In one architectural plan several references to the same parametric model may co-exist. Each such reference uses a different set of parameter values, therefore representing a unique instance-geometry. The instance-geometries are generated and refreshed automatically as needed. In the rest of this paper the mathematics behind the instance-generation process will be discussed.

\section{Displacement, Degree of Freedom, Local Shape Modification}

Most importantly our geometry must possess some degree of freedom to realize change in shape. Change in shape naturally involves change in some relative distance, which is then controlled via so called dimension-tools, discussed later in this paper. In order to provide maximum flexibility, the displacement degree of freedom and the dimension tools are kept quasi-independent from each other, requiring the user to take specific and separate actions to setup the possible displacements, and dimensions. In one parametric geometry the user may introduce multiple displacement DOFs, but then he/she must provide dimension control for each every one of them. This is necessary for the instance-geometry to become well determined. In this first version of our parametric geometry we allow only for edge displacements, because many of the practical shape modifications arising in architectural design can be implemented easily by this simple operation. This limitation, however, is not necessary; the method may be easily extended.

The edges or edge-lines of the nominal geometry can move in the perpendicular direction to their original orientations (sideways), meaning they remain parallel to the original orientation (Figure 1). The amount of such edge-shift is the internal degree of freedom of our parametric geometry, which is indirectly controlled by the dimension tools. Due to such indirect control, these internal DOFs remain solution variables of the equation system that we need to solve for, when generating the final geometry. In theory, each every one of the edges may define such internal DOF. In practice there will be some edges not allowed to move. Still another case is when several edges are allowed to move with the same "speed" -all the edges within this group possess the same amount of shift-. Such group-edges share exactly one internal DOF, and so the user only needs to provide one dimension-tool to control the group-displacement. The challenge of the final geometry generation is that the degrees of freedoms discussed so far are not independent from each other, as the corresponding dimension-tools are often referring to, or defined relative to parts of the geometry, which may be also displaced due to changes in some other parameter values. This interrelationship between displacement and the dimension-tools results in an interdependent and nonlinear system of equations to solve for.

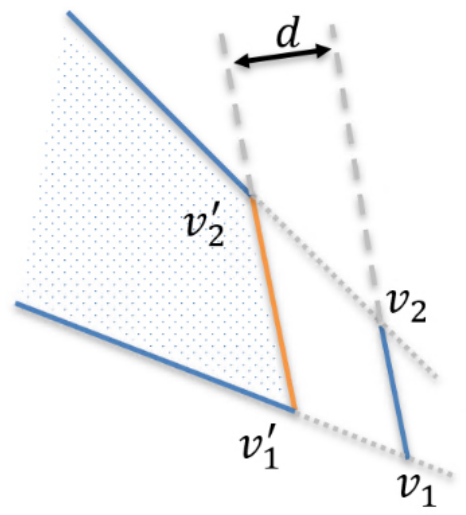

Fig. 1 Edges of the model are free to shift, remaining parallel to the original orientation $\left(v_{1}^{\prime}, v_{2}^{\prime}\right)$. The amount of displacement introduces a new variable, $d$. This is also refered as Degree of Freedom.

Because of the edge-line displacements, the edge-end vertices will be displaced as well. From the perspective of one displaced edge, its end vertex will obey two rules of the same kind: it must remain on the displaced edge-line and the edge-line of the neighboring edge, regardless if the neighboring edge-line is displaced or not. These two constraints will fully determine the two coordinate values of the vertex and must be represented by separate and independent equations in the system. Our primary goal is to determine the new coordinate values for each moving vertex. Clearly, 
the coordinate values will be our primary solution variables or unknowns along with the unknowns associated with the displacement degree of freedoms.

According to the above discussion, one displaced edge will generate two equations, one for each end-vertex (1).

$$
\begin{aligned}
& \left(\left(\boldsymbol{v}_{2}^{\prime}-\boldsymbol{v}_{2}\right) \times\left(v_{1}^{\prime}-\boldsymbol{v}_{2}\right)\right)_{z}=d\left\|\boldsymbol{v}_{2}^{\prime}-\boldsymbol{v}_{1}^{\prime}\right\| \\
& \left(\left(\boldsymbol{v}_{2}^{\prime}-\boldsymbol{v}_{1}\right) \times\left(\boldsymbol{v}_{1}^{\prime}-\boldsymbol{v}_{1}\right)\right)_{z}=d\left\|\boldsymbol{v}_{2}^{\prime}-\boldsymbol{v}_{1}^{\prime}\right\|
\end{aligned}
$$

In these equations $d$ is the internal degree of freedom of the edge. It remains a solution variable. $v_{1}^{\prime}$ and $v_{2}^{\prime}$ are the original vertex positions and therefore they are fixed values. When two displaced edges are joining via a common vertex, no extra equation is needed. However, where a non-displaced edge is the neighbor of a displaced edge, one equation is still required to keep the moving common vertex $-\boldsymbol{v}$ - on the fixed edge line $-\boldsymbol{v}_{1}^{*}, \boldsymbol{v}_{2}^{*}$ - of the non-displaced edge:

$$
\left(\left(v^{*}{ }_{2}-v\right) \times\left(v^{*}-v\right)\right)_{z}=0
$$

Here $\boldsymbol{v}$ is one of $\boldsymbol{v}_{1}$ or $\boldsymbol{v}_{2}$ in Equation (1) and it is also the vertex of the non-displaced neighbor-edge, $\boldsymbol{v}^{*}, \boldsymbol{v}^{*}{ }_{2}$ are the original and fixed locations of the non-displaced edge.

\section{Dimension tools}

The dimension control tools are nothing else, but structured distance-constraints. The distance is defined between two anchors. Each anchor is defined either by certain parts of the geometry, or by fixed points in the global coordinate system. Accordingly, anchors may be of the following types:

- Vertex anchor,

- Edge anchor,

- Point anchor -point is fixed to the global coordinate system-.

Theoretically we end up with $3 \mathrm{X} 3$ different types of dimension tools or distance constraints: Vertex-vertex, vertex-edge, etc. Not all of these types are actually used as some of these combinations obviously make no sense (e.g. Point-Point type for obvious reason). In some combinations the user may provide information for the distance metric: he/she may use scalar projection or Euclidian metric. The effect of the dimension control tool is represented by an extra equation. This equation involves the parameter value associated to the dimension tool. For example, in case of scalar projection we have:

$$
\left(\boldsymbol{\alpha}_{\text {end }}-\boldsymbol{\alpha}_{b e g}\right) \cdot \boldsymbol{v}_{s p}=p
$$

Here $\boldsymbol{\alpha}_{b e g}$ and $\boldsymbol{\alpha}_{\text {end }}$ are the expressions for the positions of vertex- or edge-anchors at the two ends of the dimension control tool, is the fixed vector of the scalar projection, and is the distance-parameter of the dimension control tool.

It is also possible to use Euclidean distance as the basis of the dimension control. The expression on the left-hand side of Equation (3) above is replaced with the formula for the Euclidean distance between $\boldsymbol{\alpha}_{b e g}$ and $\boldsymbol{\alpha}_{\text {end }}$ resulting in the below equation:

$$
\left\|\boldsymbol{\alpha}_{\text {end }}-\boldsymbol{\alpha}_{b e g}\right\|=p
$$

\section{Parameterized Geometry}

The augmentation of any geometry with the dimension tools and the displacement degree of freedoms make the geometry parameterized and ready to create new instances. An example is given in Figure 2. The geometry and the parameters are all set up, and only 4 parameters (dimension tools) are highlighted: "Top width", "Bottom Width", "Top" and "Bottom". There are 6 highlighted edges (green) with a small black arrowhead near the center of each. These are the edges that possess displacement DOF, and the arrowheads signify the positive direction of each edge-shift. Some edges here use a common DOF, so that finally the total number of DOF matches the number of free parameters (4) in this system. The two highlighted vertical edges on the top share one common DOF and similarly the two vertical highlighted edges on the bottom also share one. The horizontal highlighted edges on the top and bottom have the other two DOF in the system and they do not share these with other edges. 


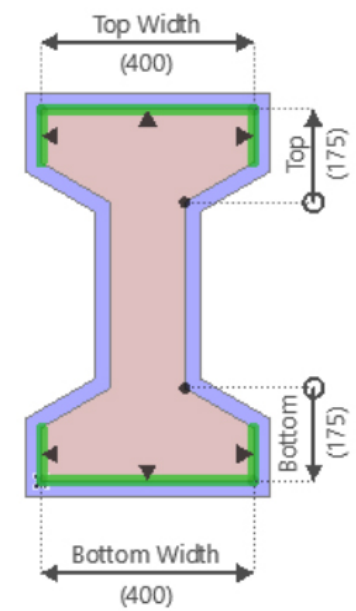

Fig. 2 Example geometry: "I" beam profile with parameterization of the bottom and top part. The parameters are defined by the four dimension arrows, showing the name and the actual/nominal value of the parameter. The arrows are anchored to reference vertices and/or edges. Please note, that due to changes in parameter values both anchor-objects of a dimension arrow may be displaced. Anchor-object displacement is possible due to the Edge DoF-s discussed earlier.

\section{The Equation System}

The equations presented so far only serve as illustration and to highlight the main idea behind our parametric geometry engine. For example, Equation (2) is useless and needs to be replaced in case of circular arc type non-displaced edges. It is not our goal to document all these small details, but please note, that the equations are small, simple and acting on only a few geometric parts of the entire geometry. For this reason, it is easy to extend the system with new types of equations, as they fit easily into the information management framework of the engine.

It is worth to mention, that due to the small, locally acting equations, the entire equation system will be sparse, and therefore allows for fast solution methods.

Our parametric geometry engine is responsible to put together the proper equation system for the given problem. This is done by appropriate interpretation of the incoming data, consisting of the nominal geometry and the setup for the parametric control. This is a complex task by itself and it is crucial for the successful engine operation, nevertheless, it is not the subject of this paper.

\section{Solution process}

For a given problem we need to solve for a set of equations as discussed above. The equations are transformed to residuals by simply forming differences from the left and corresponding right-hand sides. The residuals are then properly weighted. Each residual-implementation incorporates the weighting and selects from two possible weight factors, according to its type. Residuals evaluating to have measurement units of $[\mathrm{m}]$ select $w_{1}$ and those, evaluating to have units of $\left[m^{2}\right]$ select $w_{2}$ :

$$
\begin{aligned}
& \boldsymbol{w}_{1}=\frac{4}{p} \\
& \boldsymbol{w}_{2}=\frac{1}{\alpha}
\end{aligned}
$$

Here $p$ is the perimeter and $\alpha$ is the area of the bounding rectangle around the nominal geometry. This weighting is chosen to make sure that the residuals of different types become unitless and normalized. We expect that at the end of the solution process, the residuals become zero, and for this reason the weight values do not influence the final solution of the problem as it would be in case of finite residual-value solutions. However, it is still important to choose proper weights to ensure fast practical rate of convergence during the solution process.

The solution of the problem is found by minimization of the sum of squared residuals $\sum_{i} r_{i}^{2}=\boldsymbol{r}^{T} \boldsymbol{r}$. This is done pretty much the way as described in standard textbooks for optimization algorithms. For the sake of reference, I repeat here the fundamental derivation of the step-equation, following [1]: 


$$
\begin{gathered}
\boldsymbol{A}=\left[\begin{array}{lll}
\nabla r_{1} & \ldots & \nabla r_{m}
\end{array}\right] \\
\boldsymbol{A}=\nabla\left(\boldsymbol{r}^{T} \boldsymbol{r}\right)=\mathbf{2} \boldsymbol{A} \boldsymbol{r} \\
\boldsymbol{H}=\mathbf{2} \boldsymbol{A} \boldsymbol{A}^{\boldsymbol{T}}+2 \sum_{i} r_{i} \nabla^{2} r_{i}
\end{gathered}
$$

Here $\boldsymbol{A}$ is the matrix of first derivatives of the residuals, $\boldsymbol{g}$ is the first derivatives of $\sum_{i} r_{i}^{2}$ and $\boldsymbol{H}$ is the second derivatives of $\sum_{i} r_{i}{ }_{i}$. During the solution process a step in the optimization variables needs to be taken to improve the current iterate, so we need to solve the below equation:

$$
\boldsymbol{H s}=-\boldsymbol{g}
$$

Instead of solving Equation (7) in each iteration, we drop the second order terms [1] and arrive to a much simpler form:

$$
2 A A^{T} S=-2 A r
$$

Or

$$
A A^{T} S=-A r
$$

One reason for the above simplification is that we expect our problems to have zero residuals at the exact solution, so near the solution the terms $r_{i} \nabla^{2} r_{i}$ are expected to be small. Dropping these small terms has minor effect on the accuracy of our matrix of second derivatives, therefore still allowing for fast, second order convergence. Because of this simplification we only need to calculate the first derivatives of each residuals. We have developed a computational library to support calculations of any quantity with exact (analytic) first order derivatives [4]. This is simply done in $\mathrm{C}++$ : We have introduced a new $\mathrm{C}++$ class, which represents a real valued quantity and its first order derivatives, and we have built an entire arithmetic over this new type via mostly operator-overloading (see $\mathrm{C}++$ ) in such a way, so that each result-object is also receiving the proper derivative information and propagates it into consecutive operations until the calculation is finally done. Implementing the residual calculations entirely with this new value-type and the new arithmetic, the derivatives of the residual values are automatically available at the end of each calculation. Please note, that the elements of $\boldsymbol{r}$ and $\boldsymbol{A}$ are the residual values and their derivatives respectively, and so at the end of residual calculations, these matrices can be populated immediately from the new value-type we have just discussed.

During the solution process Equation (9) is solved several times for the actual step-proposal, followed by the improvement of the solution-estimate along the step -line-search process ([1], Page 33)-. This process of repeated improvements supposed to lead to the final solution of the problem, which then will satisfy some convergence criteria, mostly based on reasonable geometrical tolerances. The solution for the step is done with Conjugate Gradients (CG) iteration [2], but the matrix operations required in the CG algorithm, such as $\boldsymbol{M p}$ and $\boldsymbol{p}^{\boldsymbol{T}} \boldsymbol{M p}$ products are implemented in the sparse form: $\boldsymbol{A} \boldsymbol{A}^{T} \boldsymbol{p}$ and $\boldsymbol{p}^{\boldsymbol{T}} \boldsymbol{A} \boldsymbol{A}^{\boldsymbol{T}} \boldsymbol{p}$, where the $\mathrm{CG}$ is applied to the equation $\boldsymbol{M x}=\boldsymbol{b}$ and $\boldsymbol{M}=\boldsymbol{A} \boldsymbol{A}^{\boldsymbol{T}}, \boldsymbol{b}=-\boldsymbol{A r}$.

\section{Continuation}

The nominal geometry is our initial approximation for the final solution of our problem. The solution process may be therefore viewed as the transformation or distortion of the nominal geometry to the final one via repeated improvements. This process is augmented with a technique to prevent it finding a local optimum, while ensuring fast convergence all the time. Ultimately our goal is to make sure that neglecting the term $r_{i} \nabla^{2} r_{i}$ will yield always an accurate-enough Hessian matrix. One way of achieving this is to reduce the residual values right at the beginning of the search process and keep them reduced during the entire process. Modification of our residuals, however, means that the original optimization problem is modified. For this reason, the modification should be such that at the end of the solution process, we still solve for the original problem. Ultimate reduction of the residuals would be to make sure they all have zero values. At the beginning of the process this could be achieved by simply replacing the parameter goal-values with the known parameter values belonging to the nominal or initial geometry. On the other hand, at the end of the process the parameter-values must resume those of the original problem. Following this logic, during the optimization process the parameter goal-values are substituted with new expressions continuously changing the actual parameter values from those belonging to the initial geometry to the goal-values provided by the user:

$$
p^{*}=C\left(\lambda, p_{\text {goal }}, p_{\text {initial }}\right)
$$


Where on the right-hand side the expression for $C$ should be a simple formula, using the initial and final (goal) parameter values and $\lambda$, which is a new auxiliary parameter, sweeping from 0 to 1 , and $p^{*}$ is the replaced parameter goal-value. $C$ should have the following properties:

- $C\left(0, p_{\text {goal }}, p_{\text {initial }}\right)=p_{\text {initial }}$

- $C\left(1, p_{\text {goal }}, p_{\text {initial }}\right)=p_{\text {goal }}$

- $C$ should be continuous and monotonic function of $\lambda$

For example, the simplest form of such expression is:

$$
C\left(\lambda, p_{\text {goal }}, p_{\text {initial }}\right)=\lambda p_{\text {goal }}+(1-\lambda) p_{\text {initial }}
$$

Using the above discussed replacement of the goal values all over in the problem with the same and common $\lambda$ parameter, we just created homotopy between the original problem and the nominal problem. The later problem is obviously solved; the solution is the nominal geometry. Homotopy Continuation methods [3] are based on following the known solution of the initial problem to the solution of the final problem -our original problem-. Instead of the usual path-following prediction and correction steps of the Homotopy Continuation methods, which require some scheduling of the continuation parameter, we propose to throw the continuation parameter into the set of unknowns of the original system of equations, and use a special and simple residual, to replace the schedule for the continuation parameter:

$$
r_{\text {continuation }}:=w_{\text {continuation }}(\lambda-1)
$$

Of course, the initial estimate for $\lambda$ will be zero, so initially all the other residuals are also zero, except for $r_{\text {continuation }}$. At the solution $r_{\text {continuation }}$ should be zero, and therefor $\lambda=1$, meaning that finally we solved our original problem.

During the solution process of the equation system augmented with the continuation-parameter, continuation-residual and substitutions for the parameter goal-values, we expect that the solution for the search direction will remain well conditioned, and the line search will limit the step appropriately, limiting the step-size in continuation parameter as well. This results in an automatic feedback to control the continuation parameter, meaning that the process becomes selfadaptive. When strong nonlinearities are present in the original equation system, the adaptive-control of the continuation parameter may be advantageous, as it automatically reduces the steps in the continuation parameter value as well, and so it keeps the solution estimate close to be a solution of a problem-version between the initial and final problem.

The above described method needs and benefits from fine tuning. The proper -preferably automatic- determination of the weight used in the continuation residual is of most importance. The smaller this weight is, the slower the continuation parameter will approach one, meaning that the method becomes more conservative, more appropriate to solve problems with strong nonlinearities. This is so, because the smaller steps in continuation parameter will result in smaller steps in the other unknowns, as the steps in deforming the problem from the initial one to the final one will also be smaller. Due to the small steps in general, the solution-estimate also expected to remain closer to the current deformed problem. The need for small steps may suddenly disappear as sharp "corners" of nonlinearities are left behind, and then the quadratic nature of the convergence will resume.

In our implementation we took the simple and well working approach that is, to set $w_{\text {continuation }}$ according to the formula below:

$$
\left(w_{\text {continuation }}\right)^{2}=\left.\sum_{i} r_{i}^{2}\right|_{\lambda=1, x=x_{0}}
$$

Here $x=x_{0}$ simply means that all solution variables, except for $\lambda$, are set to the initial value (the known solution of the nominal problem), $\lambda=1$ signifies original problem without the use of any continuation. So, the right-hand side is simply the sum of squared residuals of the original problem at the initial estimate. Please note that this choice is based purely on experimentation for our particular class of problems, and still subject of research and improvements. Currently in our engine the formula above is evaluated only at the beginning of the optimization process but $w_{\text {continuation }}$ could be updated before each step-solution of the iteration. For example, one could gather information from the CG process and the linesearch or quantify the current improvement speed and establish some heuristics to modify $w_{\text {continuation }}$ accordingly. 
The continuation technique discussed above is very similar to the technique of Homotopy Continuation (HC), but it is a more relaxed version, as the correction step of $\mathrm{HC}$ is not executed at all. This simply means that during the optimization process the approximate solution will not become the solution of the current deformed problem -a problem between the initial and original problem-. For this reason, the method is more of the augmentation of a regular optimization process than path-following $\mathrm{HC}$ method. Nevertheless, it is a promising way to tackle hard to solve optimization problems.

\section{Parameter Sensitivity of the final Geometry}

In Finite Element Analysis (FEA) it is sometimes fundamental to explore the design space in terms of design parameters. These parameters can be excitation source values, boundary condition values, material property values or parameters of the device-geometry. The goal of the FEA is to determine the value of certain quantities like electromagnetic fields, acting forces or scattering parameters. Some of these quantities are the results of postprocessing the primary fields. The need for calculating the sensitivity of such quantities with respect to design parameters arise naturally for example, when design requirements demand certain values for these sensitivities. Also, when the device needs to be optimized automatically by some numerical optimization tool, it may be advantageous to make these sensitivities available for the optimization process, as they help successful optimization and support faster practical convergence. Finite differentiation techniques are commonly applied in these situations, but then this means costly and repeated FEA with re-meshing due to changes in geometry. Re-meshing introduces noise-like errors in the approximated sensitivity values, sometimes rendering these results completely useless. For the above reasons, it is worth to provide methods calculating these sensitivities without involving finite differentiation techniques, particularly for parameters impacting the geometry. Next, we will show that our methodology is perfectly capable of supporting geometric-sensitivity calculations without involving potentially harmful finite differentiations.

Our goal is to provide easy to solve equations for the sensitivities of the original solution variables with respect to a parameter $p$. Assuming, that the solution for the original parametric problem is already available, we start our derivation from the fact, that the below quantity has been minimized:

$$
\mathrm{f}_{x, p}=\sum_{i} r_{i}^{2}
$$

Here $\boldsymbol{x}$ is a vector of our original solution variables and $p$ is the parameter. We note, that there is no need for continuation in this case, so all the geometric parameters are just represented with their finite goal values. For now, let us say that we need the sensitivity with respect to only one parameter $p$. In the above sum of squares the residuals are assumed to depend on $\boldsymbol{x}$ and $p: r_{i}=r_{i}(x(p), p)$. Please note, that the solution variables $\boldsymbol{x}$ became dependent on the parameter $p$. The problem has been solved, so the gradients with respect to the solution variables are all zero, which is the first order criteria of having an extremum:

$$
\frac{\partial \mathrm{f}_{x, p}}{\partial x_{k}}=\sum_{i} \frac{\partial r_{i}^{2}}{\partial x_{k}}=\sum_{i} 2 r_{i} \frac{\partial r_{i}}{\partial x_{k}}=0 \forall k
$$

When the parameter $p$ changes, a new solution of the above equations would be required. It is expected, that for a very small change in the parameter value will result in a very small change in the solution $\boldsymbol{x}$. Following this logic, it makes sense to take the total derivative of each term $(\forall k)$ in the gradient-vector with respect to the parameter $p$ and expecting that this derivative will be zero, as the gradient itself remains zero as well:

$$
\frac{d\left(\sum_{i} 2 r_{i} \frac{\partial r_{i}}{\partial x_{k}}\right)}{d p}=0 \forall k
$$

Expanding on the left-hand side:

$$
\begin{gathered}
\frac{d\left(\sum_{i} 2 r_{i} \frac{\partial r_{i}}{\partial x_{k}}\right)}{d p}=\mathbf{2} \sum_{i} \frac{d\left(r_{i} \frac{\partial r_{i}}{\partial x_{k}}\right)}{d p}=\mathbf{2} \sum_{i}\left\{\left(\frac{d}{d p} r_{i}\right) \frac{\partial r_{i}}{\partial x_{k}}+r_{i}\left(\frac{d}{d p} \frac{\partial r_{i}}{\partial x_{k}}\right)\right\}= \\
\mathbf{2} \sum_{i}\left\{\left(\sum_{m} \frac{\partial r_{i}}{\partial x_{m}} \frac{\partial x_{m}}{\partial p}+\frac{\partial r_{i}}{\partial p}\right) \frac{\partial r_{i}}{\partial x_{k}}+r_{i}\left(\sum_{m} \frac{\partial \frac{\partial r_{i}}{\partial x_{k}}}{\partial x_{m}} \frac{\partial x_{m}}{\partial p}+\frac{\partial \frac{\partial r_{i}}{\partial x_{k}}}{\partial p}\right)\right\}
\end{gathered}
$$


Now let us introduce the notation $s_{m}=\partial x_{m} / \partial_{p}$, which is the sensitivity of the solution variable $x_{m}$ :

$$
\mathbf{2} \sum_{i}\left\{\left(\sum_{m} \frac{\partial r_{i}}{\partial x_{m}} s_{m}+\frac{\partial r_{i}}{\partial p}\right) \frac{\partial r_{i}}{\partial x_{k}}+r_{i}\left(\sum_{m} \frac{\partial^{2} r_{i}}{\partial x_{m} \partial x_{k}} s_{m}+\frac{\partial^{2} r_{i}}{\partial p \partial x_{k}}\right)\right\}
$$

Finally, please note, that $r_{i}=0 \forall i$, as we have a zero-residual problem. The second term in the summation can be left out of our formula. For nonzero-residual problems, this term would remain, and would mean that the second order derivatives of the residuals would be necessary. In our case, we end up with the simple equation:

$$
\sum_{i}\left(\sum_{m} \frac{\partial r_{i}}{\partial x_{m}} S_{m}+\frac{\partial r_{i}}{\partial p}\right) \frac{\partial r_{i}}{\partial x_{k}}=0 \forall k
$$

Obviously, the solution of Equation (19) yields the sensitivities with respect to one parameter only, and so the solution process needs to be repeated for all the remaining parameters. One can see, that if put in matrix form, the matrix multiplying our unknowns is the same for all these solutions, therefor one can use a solution process optimized for multiple right-hand sides. Moreover, the above matrix is identical to the matrix in Equation (9), so the same or similar sparse matrix technique may be used to extract the sensitivities.

Once we have all the geometric sensitivities, or derivatives of our new coordinates with respect to all the parameters, these could be used in the finite element solution process and post processing to obtain the valuable sensitivities of any analysis quantity.

\section{Results}

Parametric geometries are a great tool to quickly modify the nominal geometry in order to create instances, which satisfy different design requirements. Please see Figure 3, which is an example of simple beam elements, all created from the same parametric profile with different parametrization. This profile consists of only two geometrical regions. One is the core structure, the other is an insulation or veneer layer. The profile is set up so, that it keeps the original symmetry over the $\mathrm{Y}$ axis, no matter what parameter is changed and how much. The veneer part is set up so, that any shape change of the core structure will be regarded by the veneer as well, so that the veneer thickness will remain the same. Of course, this is only possible if the edges forming the exterior of the veneer region are displaced as a response to changes in the shape of the core region. In this example the veneer-thickness-constraints are not imposing uniform veneer thickness. Section by section it can be changed to different thickness values, including zero thickness, which makes the veneer disappear. This can be observed on some instances of Figure 3. This is, however, not necessary, there are ways to set up the profile for uniform veneer thickness as well. As it can be seen, the geometric profile is a tool, not only to make adjustments possible, but to enforce certain user defined geometrical rules as well. These rules can ensure proper instantiation of the architectural elements by disabling unreasonable configurations to be created. It also simplifies the process of editing such elements: The symmetry in our example was automatically achieved, without the need of extra user interaction.
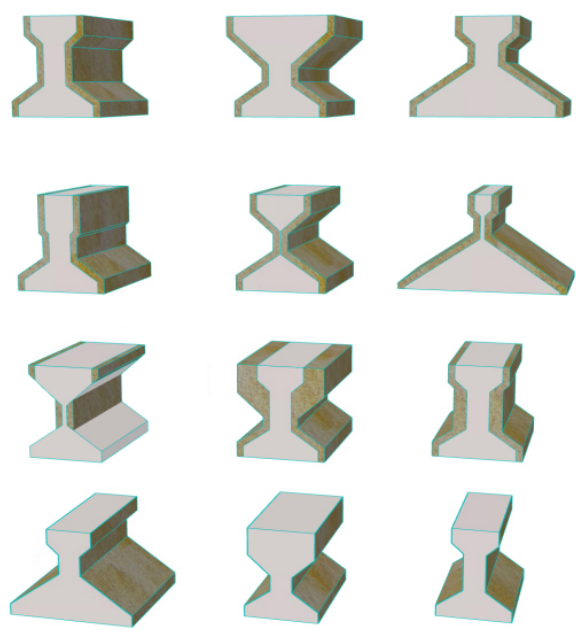

Fig. 3 Different 3D instances of "I" beams created from the same parameterized profile by applying different value combinations for the parameters. 


\section{Conclusions, Future possibilities}

The above discussed example verifies, that the proposed mathematical model for morphing 2D nominal geometries along with the robust, optimization based numerical solution process provides reasonable flexibility or -if needed- strict control over the family of geometries produced from a parameterized model. Robustness of the solution process was greatly enhanced by the newly proposed optimization-embedded continuation technique.

It is certainly true, that the only shape modification technique proposed in this paper -parallel edge displacementremains a limitation for some practical users. However, the possibility of extensions is inherent of the applied mathematical models and methods. For example, it would be easy to allow for edge rotations, or edge-shifts along trajectories of user defined shape. Trajectory based edge-displacements could be enhanced by the combination of prescribed or free edge rotations. Allowing the displacement of the user defined trajectories themselves -moreover, controlling the shape of the trajectory- could further enhance usability. Grouping multiple edges is also a way to introduce new shape modification effects. Edge groups could displace as rigid sections, or user may provide extra formulas -expressions- determining the possible change of coordinate values of vertices involved in shaping the edge group. These extra expressions could introduce a few new variables -degrees of freedoms-, which may become solution variables as explained earlier in this paper, while "external forces or dimension-constraints, distance-constraints" control the final shape and displacement of the edge group. All this would easily fit into the proposed framework due to the clean separation of controls and the shape-degree of freedoms.

Currently the final shape of the geometry is controlled via "hard" constraints, the dimension tools. For applications that are more artistic than engineering like, the possibility of using soft controls, moreover, combining them with dynamic effects (time domain extension) could also be a promising research direction. By soft we mean, that the enforcement of the distances could be replaced by artificial forces, while artificial masses, springs and dampers would balance the effect of the acting forces. Having masses and dampers in the model provides means to control the dynamic behavior -time domain- of the models in a natural way. Even in case of static models, the use of springs as artificial shape control devices in place of our dimension controls may prove to be advantageous (e.g. allowing for overdetermined criteria systems with proper control via spring stiffness coefficients).

Extension of this methodology to 3D geometries with or without the dynamic effects is also an interesting possibility.

\section{Acknowledgements}

This work was done as part of a development project at GRAPHISOFT SE. I am grateful for the opportunity and the development team I was part of. I would like to thank my bosses, Attila Rittgasszer and Gyula Faller, for their continuous support, Péter Csikvölgyi and Mihály Balla for providing valuable time for preparation of this paper. I am also grateful for Zsolt Kerecsen, VP of Product Development at GRAPHISOFT SE, for granting permission for this publication and providing valuable time for the preparation of this publication.

\section{References}

[1] Fletcher, R. Practical Methods of Optimization. 2nd ed.

[2] Conjugate gradient method. https://en.wikipedia.org/wiki/Conjugate_gradient_method

[3] Beltrán, Carlos. (2011). A continuation method to solve polynomial systems and its complexity. Numerische Mathematik. 117. 89-113. 10.1007/s00211-010-0334-3.

[4] Automatic differentiation. https://en.wikipedia.org/wiki/Automatic differentiation 